\title{
REDUCTION OF THE THIRD BOUNDARY VALUE PROBLEM BY MEANS OF HARMONIC FUNCTIONS*
}

\author{
By CLIVE R. CHESTER (Polytechnic Institute of Brooklyn)
}

1. Introduction. Let $D$ be a simply connected domain in the $x y$ plane whose boundary, $\partial D$, is analytic. Let $a(s)$ and $h(s)$ be given continuous functions of the parameter $s$ on $\partial D$. Consider the following third boundary value problem (sometimes called the Robin problem, [3], or the Churchill problem, [6]):

$$
\begin{gathered}
\nabla^{2} \phi=0 \quad \text { in } D, \\
\phi_{n}-a(s) \phi=h(s) \text { on } \partial \mathscr{D} .
\end{gathered}
$$

Here $\phi_{n}$ denotes the normal derivative of $\phi$ in an outward direction.

In [1] the theory of analytic functions of a complex variable was used to reduce (1) to a first boundary value problem. Since an analytic function of a complex variable is equivalent to two conjugate harmonic functions, it seems plausible that the method of [1] could be carried out using only harmonic functions and remaining completely in the domain of real numbers. To do this in general is not as easy as it might seem primarily because the separation of the solution obtained there into real and imaginary parts is somewhat involved. In the present paper, we shall indicate some special cases in which it is possible to instrument the ideas in [1] using only harmonic functions. In the last section we also mention a generalization of the method of complex variables to more general types of boundary value problems.

2. Reduction to a first boundary value problem. Under conditions to be formulated below, (1) can be reduced to a first boundary value problem as follows. First find a nonconstant harmonic function, $u(x, y)$, which is constant on $\partial D$. ( $u$ may have singularities.) Let $v(x, y)$ be the conjugate of $u$. Then in a neighborhood of $\partial D$, at least,

$$
\begin{aligned}
& \alpha=u(x, y), \\
& \beta=v(x, y)
\end{aligned}
$$

can be introduced as curvilinear coordinates. In these coordinates, $\partial D$ is given by $\alpha=$ constant $=\alpha_{0}$ (say). Hence $\beta$ is the running coordinate on $\partial$ D.

Since $\alpha=$ const. is the equation of $\partial \mathcal{D}$,

$$
\phi_{n}=|\nabla \alpha|^{-1}\left(\alpha_{x} \phi_{x}+\alpha_{\nu} \phi_{y}\right)=|\nabla \alpha|^{-1}\left[\alpha_{x}\left(\phi_{\alpha} \alpha_{x}+\phi_{\beta} \beta_{x}\right)+\alpha_{\nu}\left(\phi_{\alpha} \alpha_{\nu}+\phi_{\beta} \beta_{y}\right)\right] .
$$

Since $\alpha$ and $\beta$ are conjugate harmonic, this reduces to

$$
\phi_{n}=|\nabla \alpha| \phi_{\alpha} .
$$

Therefore, (1b) becomes

$$
|\nabla \alpha| \phi_{\alpha}-a(\beta) \phi=h(\beta) \text { for } \alpha=\alpha_{0},
$$

or, with

$$
\begin{gathered}
c(\beta)=|\nabla \alpha|^{-1} a(\beta) \text { and } g(\beta)=|\nabla \alpha|^{-1} h(\beta), \\
\phi_{\alpha}-c(\beta) \phi=g(\beta), \text { for } \alpha=\alpha_{0} .
\end{gathered}
$$

*Received May 24, 1966; revised manuscript received September 12, 1966. 
Since $\alpha$ and $\beta$ are conjugate harmonic, $\phi$ still satisfies the potential equation,

$$
\nabla^{2} \phi=\phi_{\alpha \alpha}+\phi_{\beta \beta}=0,
$$

where now $\alpha$ and $\beta$ are the independent variables.

If $c(\beta)$ is independent of $\beta$, then we can reduce (7) to a first boundary value problem. To do this, we first find a harmonic function $H(\alpha, \beta)$ (with singularities, if necessary) such that

$$
H\left(\alpha_{0}, \beta\right)=g(\beta) .
$$

Finding such a harmonic function means solving a first boundary value problem. Once its solution has been found, the harmonic function $\phi(\alpha, \beta)$, satisfying (7), is obtained by replacing $g$ by $H$ in (7a) and solving the resulting equation, looked at as an ordinary differential equation for $\phi$ as a function of $\alpha$. To be precise, define

$$
\phi(\alpha, \beta)=\exp (c \alpha) \int_{\alpha_{0}}^{\alpha} \exp (-c \hat{\alpha}) H(\hat{\alpha}, \beta) d \hat{\alpha}+k \exp (c \alpha),
$$

where $c$ is the (constant) coefficient in (7a) and $k$ is an arbitrary constant. Then $\phi$ is harmonic if $H$ is harmonic and $\phi$ satisfies (7a) if $H$ satisfies (8). These facts are immediately verified by differentiation. Again we emphasize that $H$ may have singularities, so that (9) may, in some cases, hold only in a sufficiently small neighborhood of $\alpha=\alpha_{0}$.

3. Examples. (1) $D=$ the upper half plane, $\partial D=$ the $x$ axis, $a(s)=a$ (constant). In this case, take $\alpha=u(x, y)=y$. Then $\beta=v(x, y)=-x$. Hence (cf. [5])

$$
\phi(x, y)=\exp (a y) \int_{\nu_{0}}^{\nu} \exp (-a \eta) H(x, \eta) d \eta .
$$

More precisely, since the boundary is given by $y=0$, we take $y_{0}=0$ and have

$$
\phi(x, y)=\exp (a y) \int_{0}^{\nu} \exp (-a \eta) H(x, \eta) d \eta .
$$

$H(x, y)$ can, of course, be written down explicitly in terms of the boundary values, $h(x)$, by means of Poisson's formula.

(2) $\partial D=$ the unit circle, $a(s)=a$ (constant). In this case, take $\alpha=u(x, y)=\log r$. Then $\beta=v(x, y)=\theta$. Hence (cf. [2])

$$
\phi(r, \theta)=r^{a} \int_{r_{0}}^{r} \rho^{-a-1} H(\rho, \theta) d \rho .
$$

If $D$ is the exterior of the unit circle, then it is natural to take $r_{0}=\infty$ and obtain

$$
\phi(r, \theta)=r^{a} \int_{\infty}^{r} \rho^{-a-1} H(\rho, \theta) d \rho,
$$

which agrees with Eq. (15) of [1].

4. Remarks on the method. As is obvious by a comparison of the two, the method of the present paper is far more restricted than that of the previous one. The primary reason for this seems to be the fact that whereas the product of two analytic functions of a complex variable is again an analytic function, the analogue is not true for two harmonic functions. The trouble, loosely speaking, is that their conjugates get into the act. Therefore the use of complex variables makes feasible the solution of a much wider 
class of problems than does the method of the present paper. The method of the present paper does show, however, why most of the authors in the various references cited in [1] were forced to restrict themselves to such highly special cases as they did.

In closing, we remark that the method of [1] is obviously not restricted to third boundary value problems. Suppose the boundary condition is of the form (for a different method of treating this $B C$, see [4, Chapt. 9])

$$
\sum_{i=1}^{m}\left[a_{i}(s) \frac{\partial^{j} \phi}{\partial n^{i}}+b_{j}(s) \frac{\partial^{j} \phi}{\partial s^{i}}\right]+c(s) \phi=h(s),
$$

where $a_{i}, b_{i}, c$ and $h$ are all analytic on the closure of $\mathfrak{D}$ and given on $\partial D$. $\partial / \partial n$ denotes the normal derivative of $\phi$ and $\partial / \partial s$ its tangential derivative. then

In [1] it was pointed out that if the equation of $\partial D$ is written in the form $\bar{z}=\omega(z)$,

$$
\frac{\partial}{\partial n}=\frac{i}{\left(\omega^{\prime}(z)\right)^{1 / 2}} \frac{d}{d z}
$$

In addition, it is easy to show that

$$
\frac{\partial}{\partial s}=\frac{1}{\left(\omega^{\prime}(z)\right)^{1 / 2}} \frac{d}{d z}
$$

Hence (10) can be written in the form

$$
\operatorname{Re}\left\{\sum_{i=1}^{m}\left[a_{i}(z)\left(\frac{i}{\left(\omega^{\prime}(z)\right)^{1 / 2}} \frac{d}{d z}\right)^{i}+b_{i}(z)\left(\frac{1}{\left(\omega^{\prime}(z)\right)^{1 / 2}} \frac{d}{d z}\right)^{i}+c\right] f\right\}=h,
$$

where $\phi=\operatorname{Re} f(z)$. If we introduce

$$
F(z)=\left[\sum_{j=1}^{m} a_{i}(z)\left(\frac{i}{\left(\omega^{\prime}(z)\right)^{1 / 2}} \frac{d}{d z}\right)^{i}+b_{i}(z)\left(\frac{1}{\left(\omega^{\prime}(z)\right)^{1 / 2}} \frac{d}{d z}\right)^{i}+c\right] f
$$

then $\operatorname{Re} F=h$ on $\partial D$, so that $F$ is the solution to a first boundary value problem. $f$ is now obtained by solving the $n$th order ordinary differential equation (12) for $f$.

\section{REFERENCES}

[1] C. R. Chester, Reduction of a boundary value problem of the third kind to one of the first kind, J. Math. Phys. 40, 68-71 (1961)

[2] R. V. Churchill, The operational calculus of Legendre transforms, J. Math. Phys. 33, 165-178 (1954)

[3] G. F. D. Duff, Partial differential equations, Univ. of Toronto Press, Toronto, 1956

[4] N. I. Muskhelishvili, Singular integral equations (translated into English by J. R. M. Radok) P. Noordhoff, N. V. Groningen, Holland, 1953

[5] H. Poritsky, On reflection of singularities of harmonic functions corresponding to the boundary condition $\partial u / \partial n+a u=0$, Bull. Amer. Math. Soc. 43, 873-884 (1937)

[6] I. N. Sneddon, Elements of partial differential equations, McGraw-Hill, New York, 1957 